\title{
An MPCA/LDA Based Dimensionality Reduction Algorithm for Face Recognition
}

\author{
Jun Huang, ${ }^{1}$ Kehua Su, ${ }^{2}$ Jamal El-Den, ${ }^{3}$ Tao Hu, ${ }^{1}$ and Junlong $\mathrm{Li}^{2}$ \\ ${ }^{1}$ The State Key Laboratory of Information Engineering in Surveying, Mapping and Remote Sensing, Wuhan University, \\ Wuhan 430072, China \\ ${ }^{2}$ School of Computer, Wuhan University, Wuhan 430072, China \\ ${ }^{3}$ School of Engineering and IT, Charles Darwin University, Darwin, NT 0909, Australia \\ Correspondence should be addressed to Kehua Su; skhemail@163.com
}

Received 10 January 2014; Revised 17 July 2014; Accepted 23 July 2014; Published 31 August 2014

Academic Editor: Yi Chen

Copyright (C) 2014 Jun Huang et al. This is an open access article distributed under the Creative Commons Attribution License, which permits unrestricted use, distribution, and reproduction in any medium, provided the original work is properly cited.

We proposed a face recognition algorithm based on both the multilinear principal component analysis (MPCA) and linear discriminant analysis (LDA). Compared with current traditional existing face recognition methods, our approach treats face images as multidimensional tensor in order to find the optimal tensor subspace for accomplishing dimension reduction. The LDA is used to project samples to a new discriminant feature space, while the $K$ nearest neighbor (KNN) is adopted for sample set classification. The results of our study and the developed algorithm are validated with face databases ORL, FERET, and YALE and compared with PCA, MPCA, and PCA + LDA methods, which demonstrates an improvement in face recognition accuracy.

\section{Introduction}

Face recognition has become a topical and timely study focus in the fields of pattern recognition and computer vision for its wide application prospect $[1,2]$. Feature extraction is the key element in face recognition. Currently, diverse recognition methods use different extraction strategies. And one of the most popular algorithms is principal component analysis algorithm (PCA), which aims to find the projected directions along with the minimum reconstructing error and then map the face dataset to a low-dimensional space spanned by those directions corresponding to the top eigenvalues [3, 4]. Traditional PCA face recognition technology can reach accuracy rate of $70 \%-92 \%$ [5]. However, it is still not fully practical.

PCA has certain limitations which result in bad adaptability in the image brightness and facial expression variety [6-9]. Under either strong bright light or weak light environments, the information of the features of the face is deficient; hence the structural information from the feature points of the face image may hardly be captured using traditional algorithms like PCA [10]. In addition, existing algorithms which are based on capturing single expressions make it difficult and challenging to capture the correct features of the same person if he changes his facial expressions. Traditional PCA fails to see the natural structure and correlation represented in data set [3], which leads to potential additional loss of compact and/or useful facial representations and will result in a higher reconstruction error rate [11].

There are many recognition proposals to address limitations of PCA presented above. In [12], Bansal and Chawla proposed normalized principal component analysis (NPCA) to improve the recognition rate. They normalized images to remove the lightening variations by applying SVD instead of eigenvalue decomposition. Pereira et al. [13] introduced a new technique which can reduce face dimensions called classmodular image principal component analysis (CMIPCA) to extract local and global information to reduce illumination effects, face expressions, and head-pos changes resulting in speed-up over PCA. In [14] Tsai showed an application of dimensionality reduction techniques, such as PCA, EM-PCA, multidimensional scaling, and locally linear embedding, to identity emotion of facial animations. But the application was not for realistic human faces. 
In our method, we decided to complement some of these limitations of PCA by adopting the MPCA algorithm together with the LDA algorithm as the basis for the study $[3,15]$. The MPCA algorithm disregards the traditional method which is based on two-dimensional data and uses instead vectors and integrates multiple face images into a high-dimensional tensor and processes data in tensor space. The advantage of this approach lies in its ability to persistently structure facial information images and consequently increases the accuracy rate when spatial relationships between pixels are considered. When the light brightness changes or facial expression changes, spatial structural information between pixels becomes particularly important.

LDA was adopted to further reduce the dimensions of samples processed by MPCA as it is capable of aggregating the samples in subspace and hence improving the face recognition rate $[16,17]$. We combine MPCA and LDA to form LDA subspace, from which both MPCA features and LDA features can be extracted.

The organization of this paper is as follows. Our proposed algorithm will be discussed in Section 2. Methodology of the approach is presented in Section 3. To demonstrate the effectiveness of the proposed method, experimental results will be shown in Section 4. Finally conclusions are drawn in Section 5.

\section{Principle of MPCA}

In computer vision, most of the objects are naturally considered as $n$ th-order tensors $(n \geq 2)$ [18]. Take Figure 1 as an example; the image matrix in (i) is a 2nd-order tensor and a movie clip, while in (ii) it is a 3rd-order tensor. Traditional techniques for subspace dimensionality reduction such as PCA could transform image matrix to vectors with high dimensionality in one mode only, which cannot meet the need of dimensionality reduction. So such techniques are unable to handle multidimensional objects well and get satisfactory results. Therefore, in order to reduce dimensionality, a reduction algorithm which can directly operate on a high-order tensor object is desirable. Twodimensional PCA (2DPCA) algorithm is proposed and developed, while researches are using dimensionality reduction solutions which represent facial images as matrices (2ndorder tensors) instead of vectors [19-22]. However, 2DPCA can only project images in single mode, which results in bad dimensionality of reduction $[3,23]$. Thus, a more efficient algorithm MPCA has been proposed to get better dimensionality reduction.

2.1. Tensor Notations and Definitions. Multilinear principal component analysis (MPCA) has been introduced in details in [3], which is used to solve the problem of gait recognition. Before describing MPCA, the notations will be shown in this paper.

Vector $\alpha$ denotes 1st-order tensor. Matrix $A$ denotes 2 ndorder tensor. $A_{i j k}$ denotes 3 rd-order tensor. Higher-order tensors are indicated by $A_{i_{1} i_{2} \ldots i_{n}}$. Assume image matrix is indicated by $X \in R^{n_{1} \times n_{2}}$. Tensor space is indicated by $R^{n_{1}} \times R^{n_{2}}$. $\left(u_{1}, u_{2}, \ldots, u_{n}\right)$ indicates the orthonormal bases of vector space $R^{n_{1}}$ and $\left(v_{1}, v_{2}, \ldots, v_{n_{2}}\right)$ indicates the orthonormal bases of vector space $R^{n_{2}}$. Vector $u_{i} v_{j}^{T}$ indicates orthonormal bases of tensor space $R^{n_{1}} \otimes R^{n_{2}}$. Image matrix $X$ equals

$$
X=\sum_{i j}\left(u_{i}^{T} X v_{j}\right) u_{i} v_{j}^{T} .
$$

Define two matrices $U=\left[u_{1}, u_{2}, \ldots, u_{m_{1}}\right] \in R^{n_{1} \times m_{1}}$ and $V=\left[v_{1}, v_{2}, \ldots, v_{m_{1}}\right] \in R^{n_{2} \times m_{2}}$. Assume $u, v$ indicate subspace of space $R^{n_{1}}, R^{n_{2}}$ formed by basis vectors $\left\{u_{i}\right\}_{i}^{m_{1}}=1$ and $\left\{v_{j}\right\}_{j}^{m_{2}}=1$. Then $u \otimes v$ indicates subspace of tensor space $R^{n_{1}} \otimes R^{n_{2}}$. The result of 2 nd-order tensor $X \in R^{n_{1} \times n_{2}}$ projected to $u \otimes v$ is indicated by

$$
Y=U^{T} X V \in R^{m_{1} \times m_{2}} .
$$

Based on different objective functions, transformation matrices $U$ and $V$ can be obtained by iteration; hence dimension reduction can be achieved.

2.2. Principle of MPCA. MPCA is developed based on the PCA algorithm. Its advantage is that it operates on tensor, replacing the traditional algorithms which transform highdimensional data into one-dimensional vector. For example, to process 100 face images with size $112 \times 92$, PCA treats them as a $100 \times 10304$ matrix while MPCA treat them as a $100 \times 112 \times 92$ tensor. MPCA have the advantage of taking into account correlation in the original data which is ignored by PCA.

Assume there are tensor sets of images $\left\{X_{1}, X_{2}, \ldots, X_{M}\right\}$; a tensor object is denoted by $X_{m} \in R^{I_{1} \times I_{2} \times \cdots \times I_{N}} ; I_{n}$ denotes dimensionality of $n$-order tensor. Each tensor can be unfolded as

$$
X=S \times{ }_{1} U^{(1)} \times{ }_{2} U^{(2)} \times \cdots \times{ }_{N} U^{(N)} .
$$

Here, $U^{(n)}$ denotes orthogonal matrix. So, $\|X\|_{F}^{2}=\|S\|_{F}^{2}$ [24]. Decompose this matrix; we can get

$$
\begin{array}{r}
X_{(n)}=U^{(n)} \cdot S_{(n)} \cdot\left(U^{(n+1)} \otimes U^{(n+2)} \otimes \cdots \otimes U^{(N)}\right. \\
\left.\otimes U^{(1)} \otimes U^{(2)} \otimes \cdots \otimes U^{(n-1)}\right)^{T} .
\end{array}
$$

The key point of MPCA algorithm is to find a tensor subspace which can catch the variety of tensor objects and extract features of object. According to (4), projection of tensor samples onto tensor subspace is defined as

$$
Y=X \times{ }_{1} \widetilde{U}^{(1)^{T}} \times{ }_{2} \widetilde{U}^{(2)^{T}} \times \cdots \times{ }_{N} \widetilde{U}^{(N)^{T}}
$$

where $Y$ denotes tensor after projection. $Y=\left\{Y_{1}, Y_{2}, \ldots, Y_{M}\right\}$, $Y_{m} \in R^{P_{1} \times P_{2} \times \cdots \times P_{N}}$. Figure 2 depicts the process.

As Figure 2 shows, by projecting each mode of facial tensor $X$, low-dimensional facial tensor which satisfies maximum variance can be achieved. 


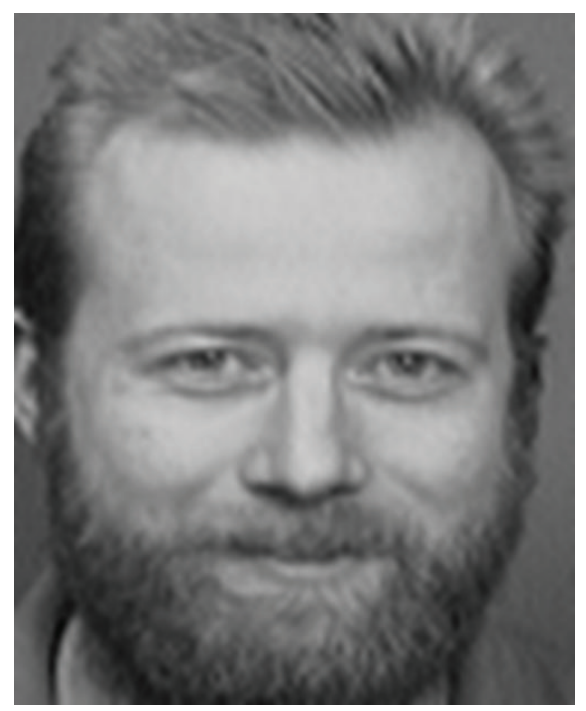

(i) Second-order tensor

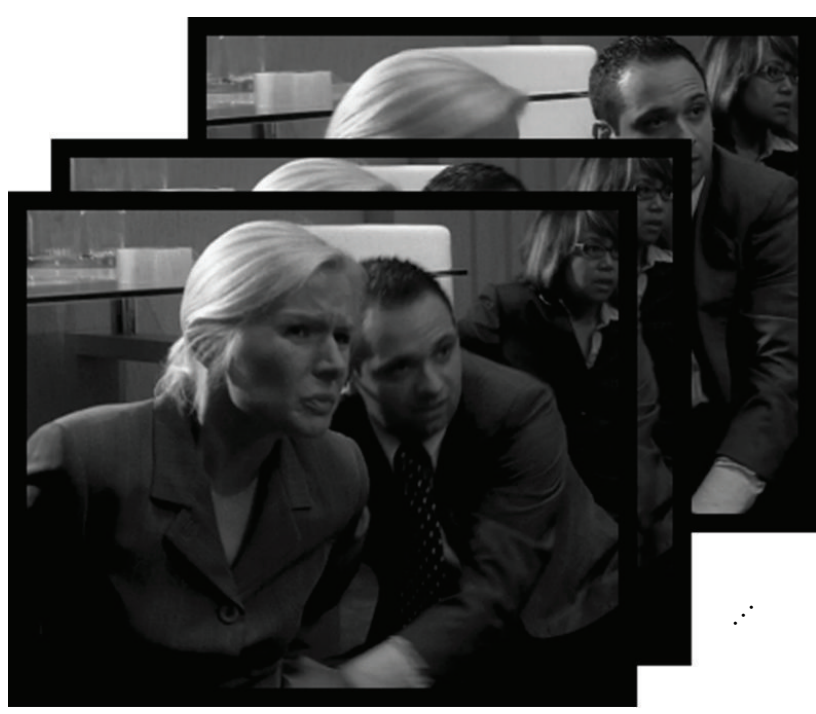

(ii) Third-order tensor

FIGURE 1: 2nd-order and 3rd-order tensor representations samples.

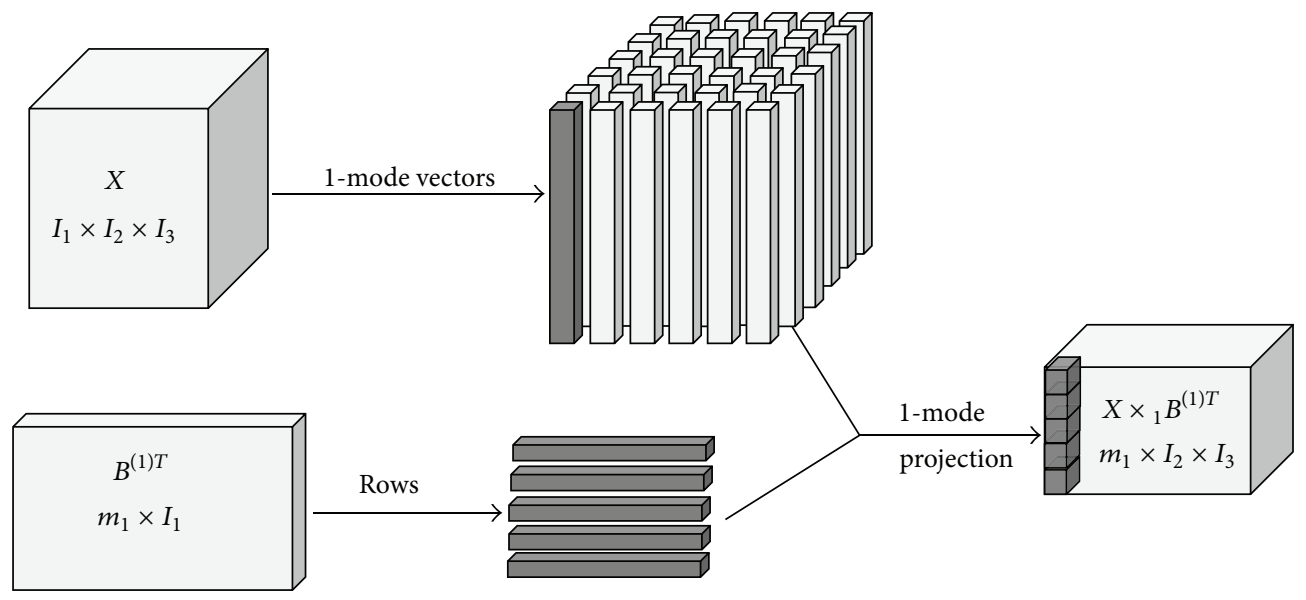

FIGURE 2: Illustration of the multilinear projection in the 1-mode vector space.

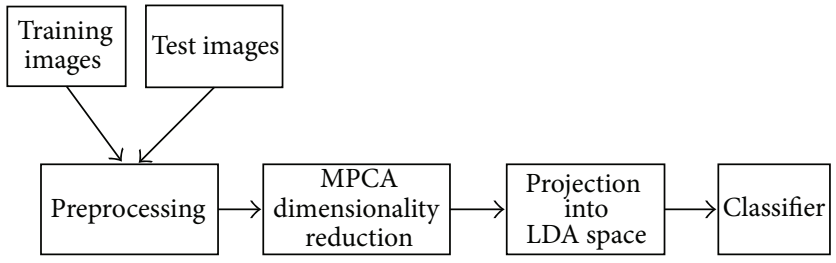

FIGURE 3: Flow chart of face recognition algorithm.
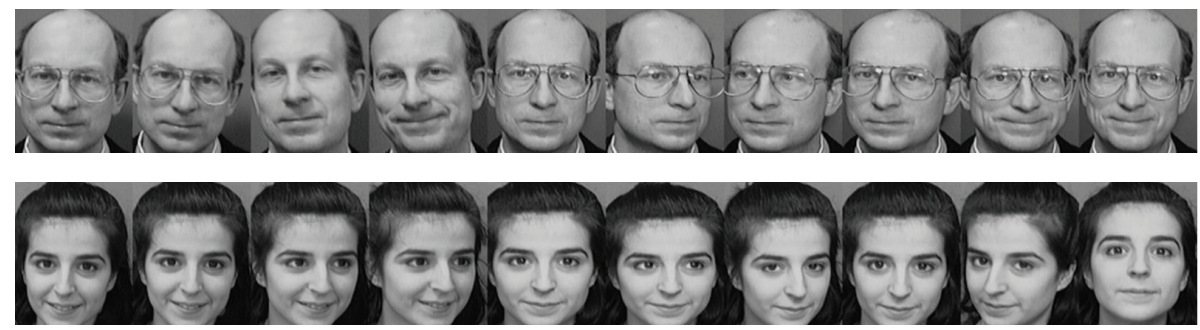

FIGURE 4: Face image examples of two persons in ORL face database. 


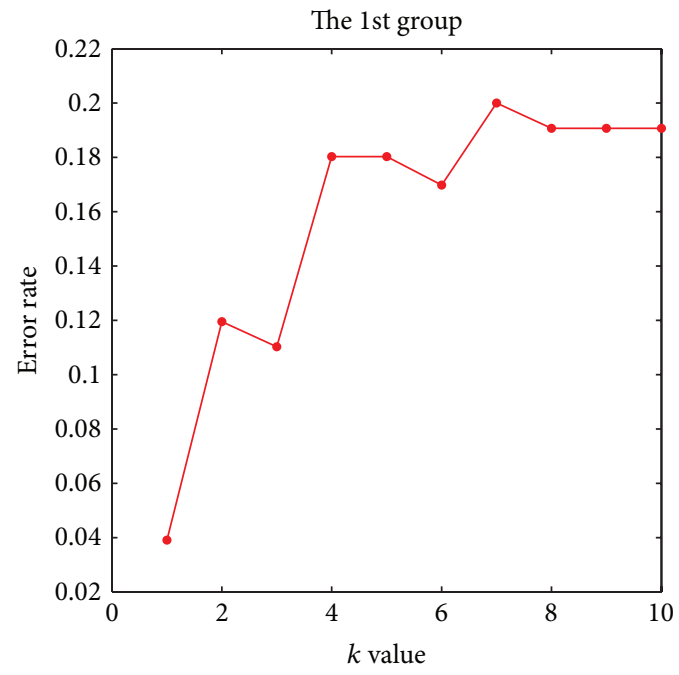

(a)

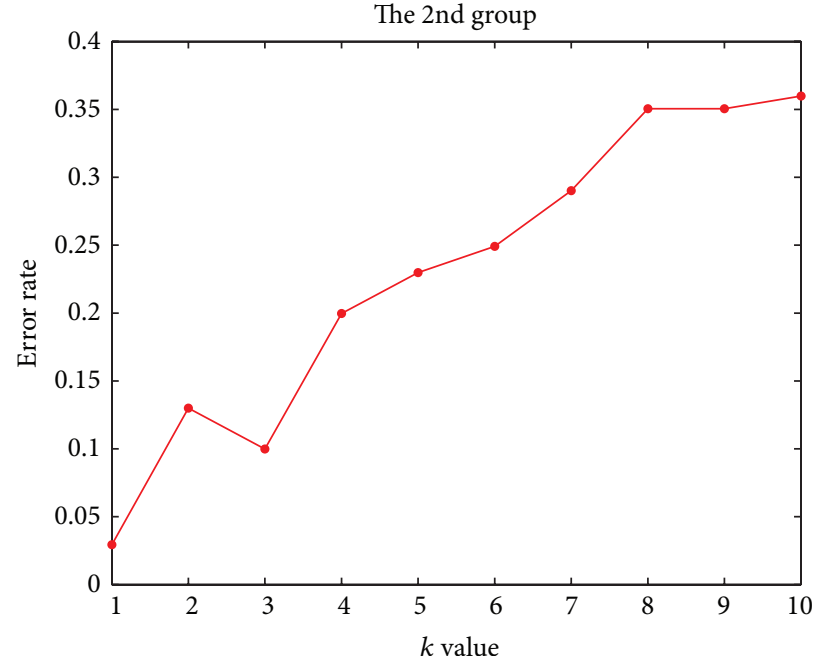

(b)

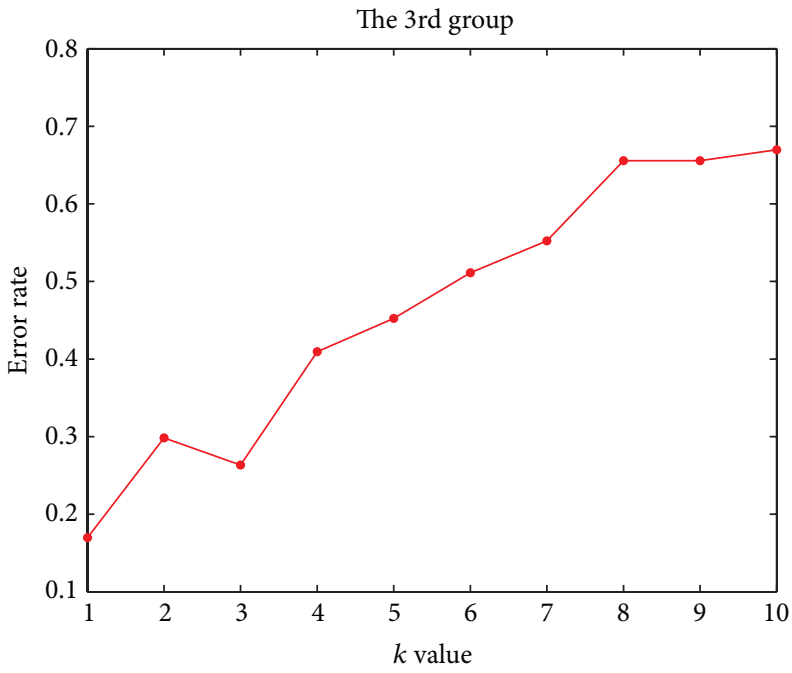

(c)

Figure 5: Recognition error rate of PCA against different $k$ values (best LDA dimension reduction). In the 1st group, $k$ equals 18 . In the 2 nd group, $k$ equals 98. In the 3rd group, $k$ equals 61 .

For tensor objects of image samples, the variance before projection is as follows:

$$
\begin{gathered}
\Psi_{X}=\sum_{m=1}^{M}\left\|X_{m}-\bar{X}\right\|_{F}^{2}, \\
\bar{X}=\left(\frac{1}{M}\right) \sum_{m=1}^{M} X_{m} .
\end{gathered}
$$

And the tensors after projection satisfy the following equation:

$$
\Psi_{Y}=\sum_{m=1}^{M}\left\|Y_{m}-\bar{Y}\right\|_{F}^{2},
$$

$$
\bar{Y}=\left(\frac{1}{M}\right) \sum_{m=1}^{M} Y_{m} .
$$

By combining (5) and (6), we can get the following equation:

$$
\begin{aligned}
\psi_{Y}=\sum_{m=1}^{M} \| & X_{m} \times \widetilde{U}^{(1)^{T}} \times \widetilde{U}^{(2)^{T}} \times \cdots \times \widetilde{U}^{(N)^{T}} \\
& -\bar{x} \times \widetilde{U}^{(1)^{T}} \times \widetilde{U}^{(2)^{T}} \times \cdots \times \widetilde{U}^{(N)^{T}} \|_{F}^{2}
\end{aligned}
$$

The MPCA algorithm equals to the resolving optimization problem:

$$
\left\{\widetilde{U}^{(1)}, n=1,2, \ldots, N\right\}=\arg \max \psi_{Y} .
$$




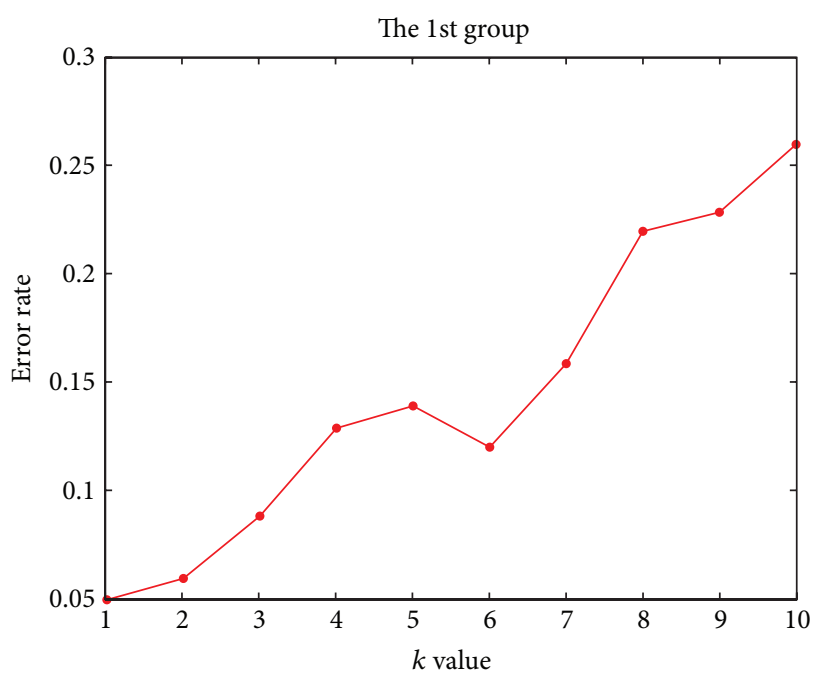

(a)

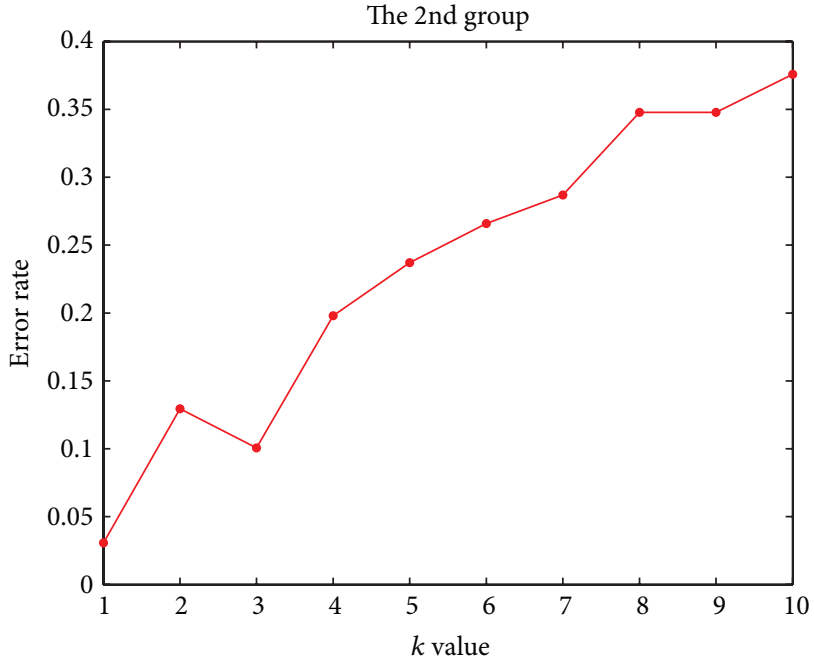

(b)

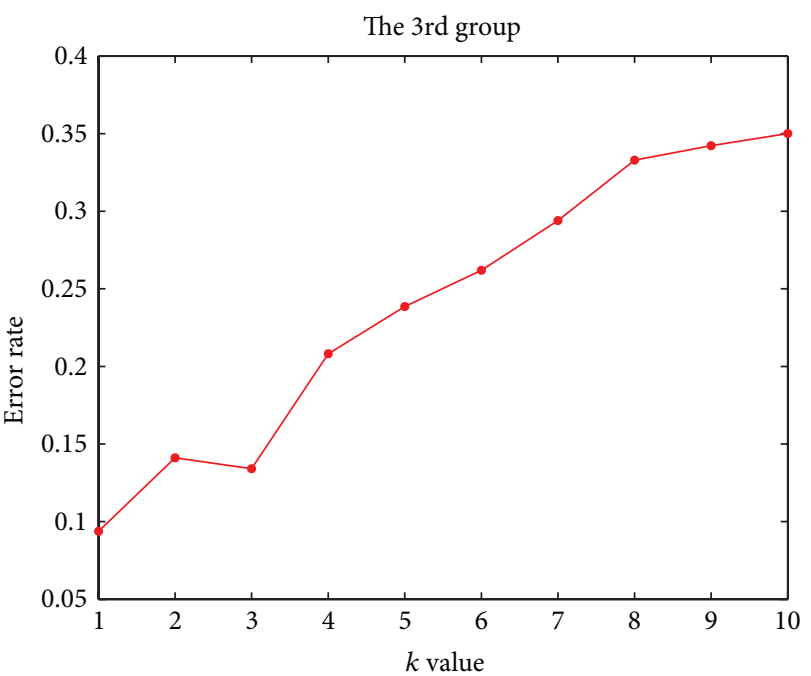

(c)

FIGURE 6: Recognition error rate of MPCA against different $k$ values.

In (9), by using alternating-least-square method (ALS), we are able to calculate local optimization procedure. When solving the $n$th projection matrix $\widetilde{U}^{(n)}$, other matrices were set constant; tensor $X$ is projected to tensor space $\left(R_{1}, \ldots, R_{n-1}, R_{n+1}, \ldots, R_{N}\right)$, where $\widetilde{U}_{j+1}^{(n)}=X \times \widetilde{U}_{j+1}^{(1)^{T}} \times \cdots \times$ $\widetilde{U}_{j+1}^{(n-1)^{T}} \times \widetilde{U}_{j+1}^{(n+1)^{T}} \times \widetilde{U}_{j+1}^{(N)^{T}}$.

Column of $\widetilde{U}^{(n)}$ can be obtained from orthogonal basis of projection subspace. Sample $X_{m}$ in (8) is projected to lower dimensional tensor $Y_{m} \in R^{P_{1} \times P_{2} \times \cdots \times P_{n-1} \times I_{n} \times P_{n+1} \cdots \times P_{N}} . Y_{m}^{(n)}, n$ thmode unfolding matrix of $Y_{m}$, is inputted to get PCA. It equals to

$$
\arg \max \sum_{m=1}^{M}\left\|\widetilde{U}^{(n)^{T}} Y_{m}^{(n)}-\widetilde{U}^{(n)^{T}} \bar{Y}^{(n)}\right\| .
$$

2.3. MPCA Algorithm. MPCA have managed to handle multidimensional objects. According to the above sections, pseudocode for the computation of the MPCA algorithm can be concluded [25] as shown in Figure 3.

Step 1. Input sample images and center them as $\left\{x_{n} \in\right.$ $\left.R^{P_{1} \times P_{2}}, n=1, \ldots, N\right\}$.

Step 2. Obtain the total scatter matrix's eigendecomposition.

Step 3. Calculate the eigenvectors and their corresponding most significant eigenvalues, and the result is output as $\widetilde{U}^{(n)}$.

Step 4. (i) Get $\left\{\widehat{y}_{n}=\widetilde{U}^{(1)^{T}} \times \widetilde{x}_{n} \times \widetilde{U}^{(2)}, n=1, \ldots, N\right\}$.

(ii) Calculate $\psi_{Y_{0}}=\sum_{n=1}^{M}\left\|\widehat{y}_{n}\right\|_{F}^{2}$.

(iii) For $k=1: K$, 


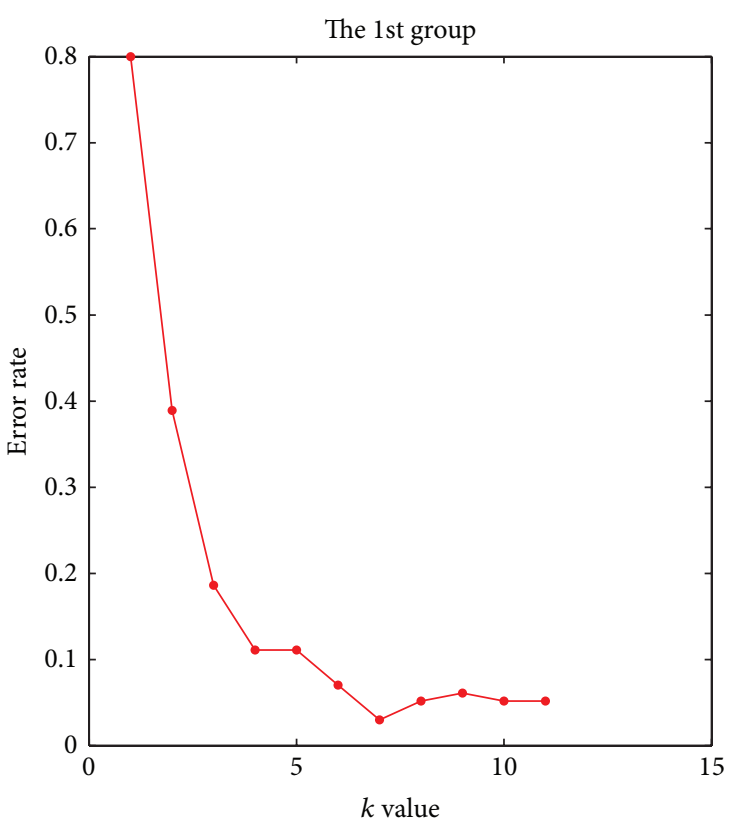

(a)

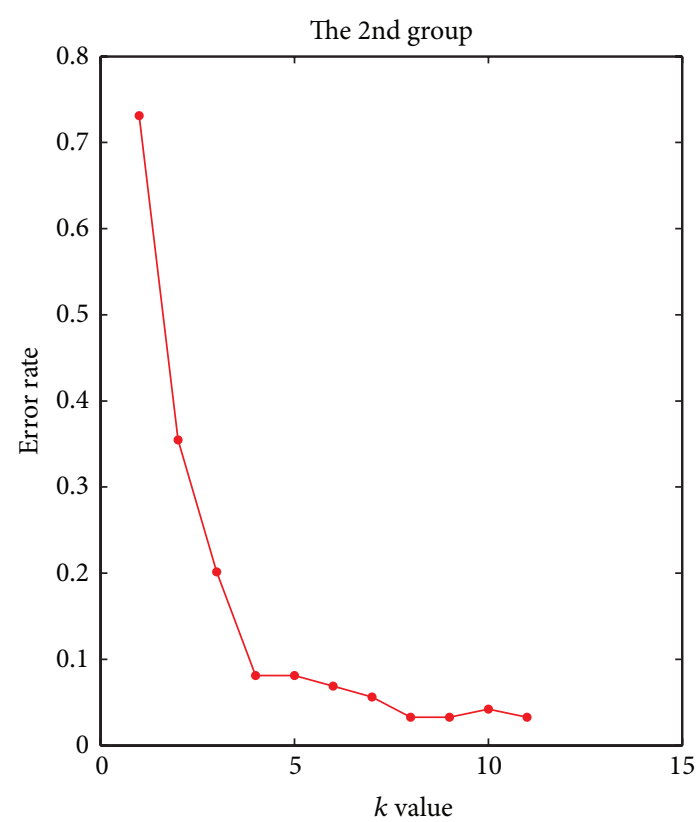

(b)

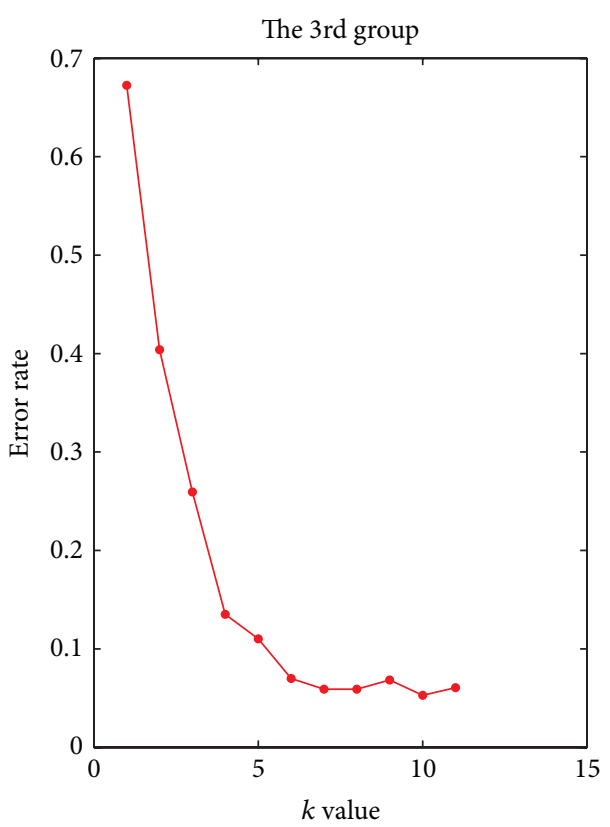

(c)

FIGURE 7: Recognition error rate of PCA + LDA against different LDA dimension reduction values.

(a) calculate the total scatter matrix's eigenvectors and their corresponding most significant eigenvalues, and the result is output as $\widetilde{U}^{(j)}$, for $j=1,2$;

(b) get $\psi_{Y_{k}}$ and $\left\{\widehat{y}_{n}, n=1, \ldots, N\right\}$;

(c) if $\psi_{Y_{k}}-\psi_{Y_{k-1}}<j$, then break the loop and go to Step 5 .
Step 5. Finally calculate the feature matrix; see the following equation:

$$
y_{n}=\widetilde{U}^{(1)^{T}} \times \widetilde{x}_{m} \times \widetilde{U}^{(2)}, \quad n=1, \ldots, N .
$$

2.4. LDA Algorithm. LDA (linear discriminant analysis) projects image onto a lower-dimensional vector space to achieve maximum discrimination as follows. 


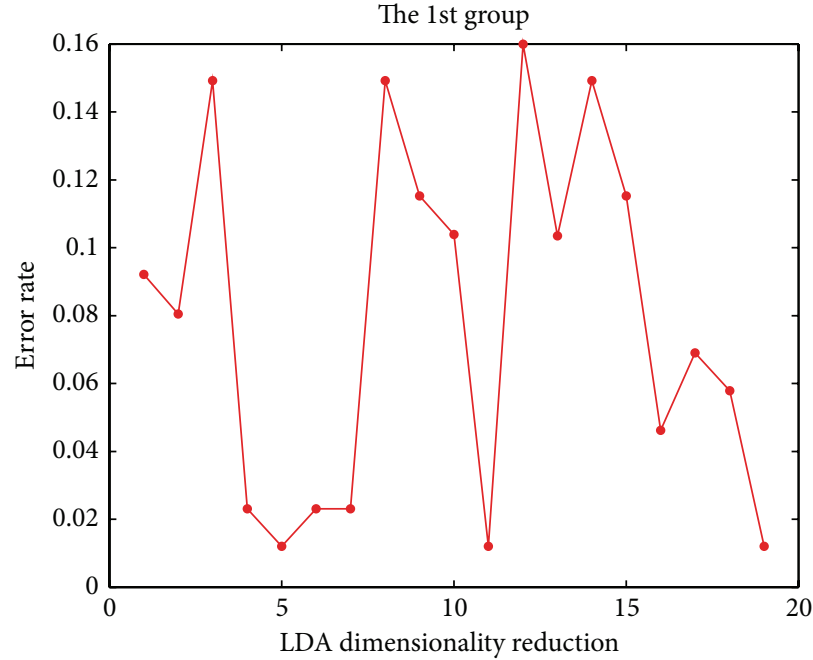

(a)

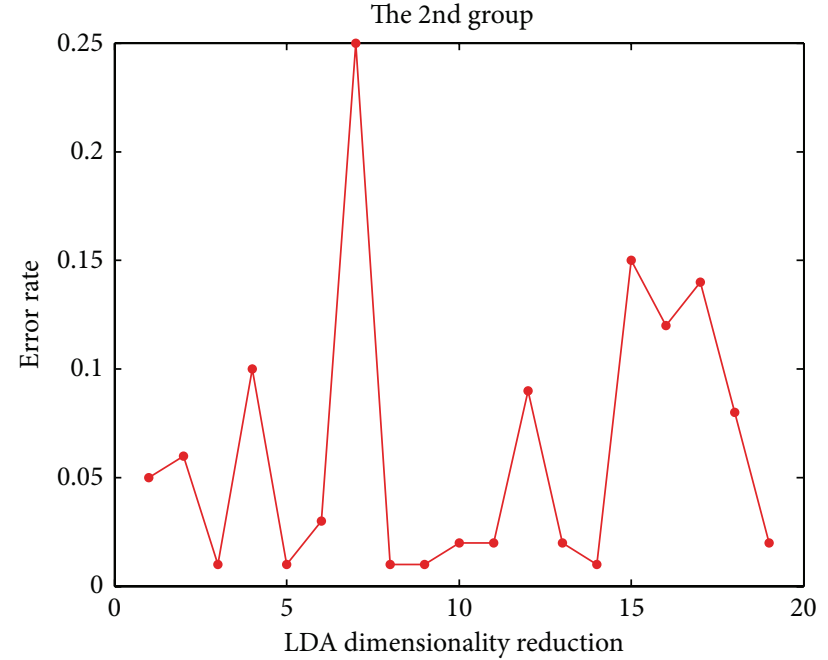

(b)

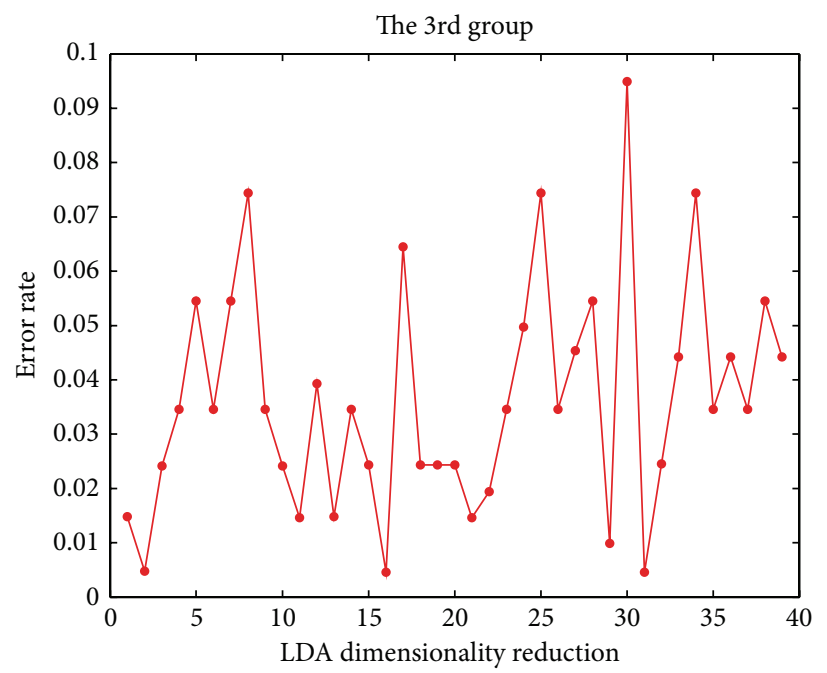

(c)

FIGURE 8: Error rate of MPCA + LDA algorithm against different LDA dimension reduction values.

Step 1. Compute the average sample values for different kinds of facial images in the original space. Total number is denoted by $c$. $X_{i j}$ denotes the $j$ th object of the $i$ th class of samples:

$$
\begin{gathered}
\overline{m_{i}}=\frac{1}{n_{i}} \sum_{j=1}^{n_{1}} X_{i j}, \quad X_{i j} \in R^{d}, \quad i=1,2, \ldots, c, \\
m=\sum_{i=1}^{c} p_{i} m_{i} .
\end{gathered}
$$

Step 2. Compute covariance matrix of each class:

$$
c_{i}=\frac{1}{n_{i}} \sum_{j=1}^{n_{i}}\left(X_{i j}-m_{i}\right) \cdot\left(X_{i j}-m_{i}\right)^{T} .
$$

Step 3. Compute within-class and between-class scatter matrices:

$$
\begin{gathered}
C_{b}=\sum_{i=1}^{c} p_{i}\left(m_{i}-m\right) \cdot\left(m_{i}-m\right)^{T}, \\
C_{w}=\sum_{i=1}^{c} c_{i} .
\end{gathered}
$$

Step 4. Compute eigenvectors of matrix $C_{w}^{-1} C_{b}$ to get projection vectors. Then dimensionality reduction data can be obtained by projection [26, 27].

After dimensionality reduction using MPCA, the matrices are arranged in columns into vectors as inputs to the LDA algorithm. By using MPCA algorithm to reduce the dimension of the image, we not only solved the problem of 


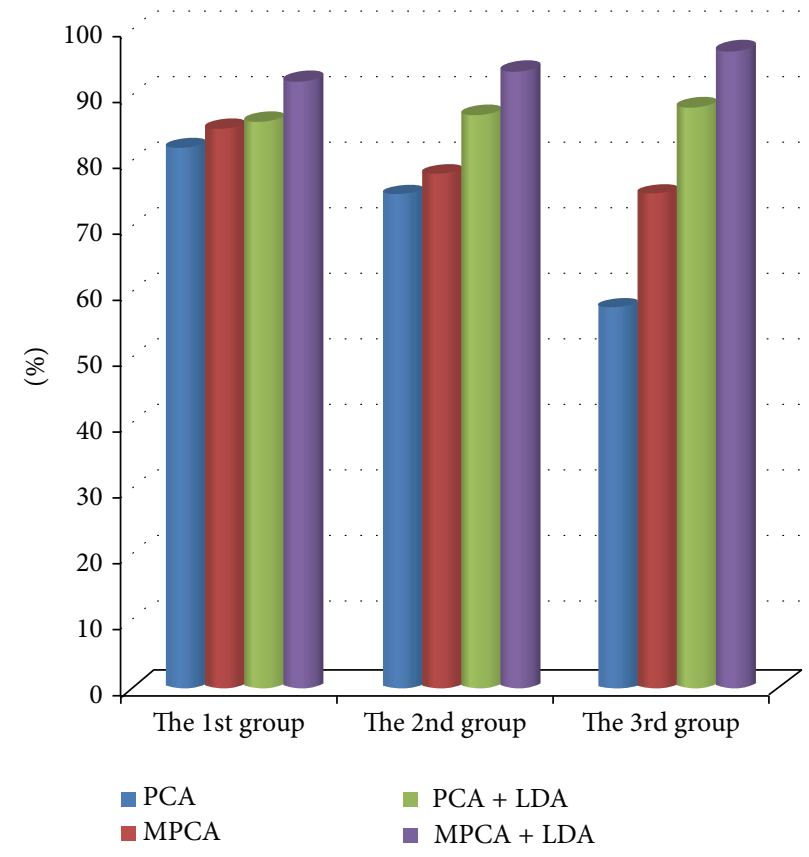

FIGURE 9: Histogram of recognition results in experiments.

singular matrix but also retained structure information in the images and thus improve the recognition rate.

2.5. KNN Algorithm. K-nearest neighbor (KNN) algorithm $[28,29]$ is adopted for sample set classification here, and the concrete steps are as follows.

Step 1. Select different parameters of $K$ value.

Step 2. Adopt the method of cross-validation on training face images, for $k=1: K$.

Step 3. Make the cross-validation error classification rate minimization and get its corresponding parameter $k$.

Step 4. Construct a prediction model with $k$.

\section{Process of the Recognition Algorithm}

3.1. Preprocessing. Image preprocessing and normalization are vital for face recognition systems as images are often affected by image quality, illumination, face rotation, facial expression [8, 30], and so forth. In order to offset above factors, it is necessary for us to carry out face normalization before facial feature extraction.

Our data is preprocessing normalized images with a resolution of $80 \times 80$. In our research, histogram equalization was applied (see (15)):

$$
y^{\prime}=\frac{y-\min (y)}{\max (y)-\min (y)} .
$$

3.2. Dimensionality Reduction Using MPCA and Feature Matrix Extraction Using LDA. MPCA reduces dimensions of input face images and generates feature projection matrix [30] that are then taken as input samples to LDA. MPCA and LDA combination were used to construct LDA subspace, from which both MPCA features and LDA features can be extracted.

The detailed steps have been described in Sections 2.2 and 2.3.

3.3. Face Recognition Using L2 Distance Measure. We used resultant output acquired above as input samples for training and applied aforesaid techniques to get the feature matrix. Then we carried out a similarity measure on image samples. In our research, we choose L2 distance for measures (see (16)):

$$
d(a, b)=\sqrt{\sum_{h=1}^{H}[a(h)-b(h)]^{2}} .
$$

KNN classifier [31] is adopted for sample set classification here, while the procedure and details are introduced in Section 2.4.

The overall approach of face recognition proposed in this paper is shown in Figure 3.

\section{Experiments}

We evaluated the performance of our algorithm based on MPCA + LDA in this research and compared with the PCA, MPCA, and PCA + LDA algorithm by performing experiments on ORL databases [32]. In order to examine the ability of our method, we also try it on other classical face databases such as FERET and YALE. 

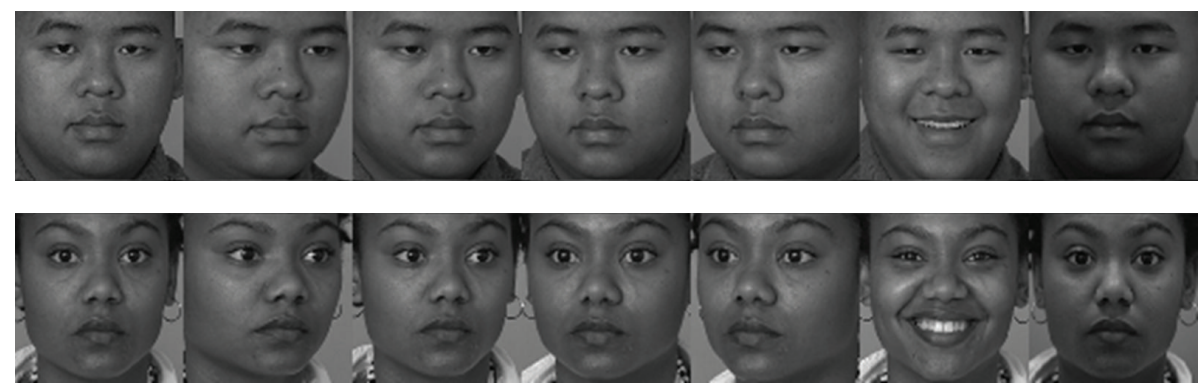

FIGURE 10: Face image examples of two persons in FERET face database.

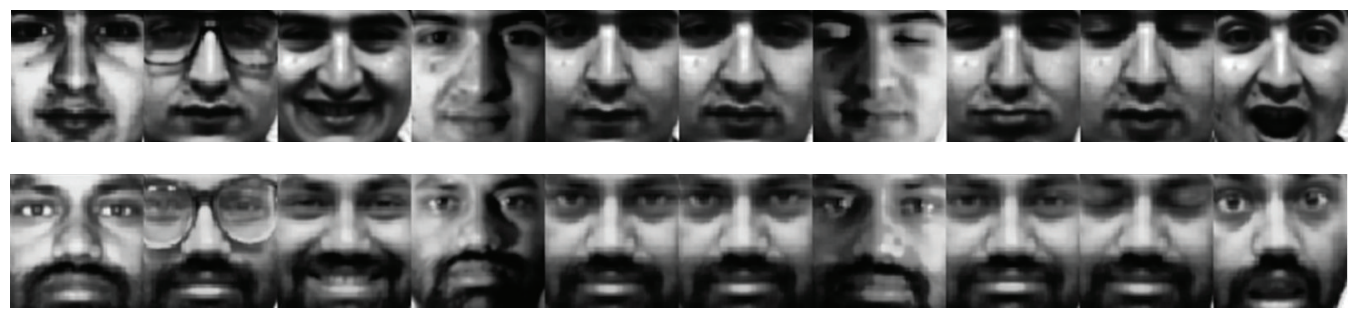

FIGURE 11: Face image examples of two persons in YALE face database.

The experiments were conducted with three groups. We choose part of images in each group for training, while the rest for testing. As the probabilities for each kind of facial samples are the same, then $P_{i}$ that equals 1 is set in LDA algorithm.

Initially, we tested how different parameters affect the recognition error rate and how classification result is affected by dimensionality using the MPCA, dimensionality after using the LDA and $k$ value of KNN algorithm. LDA algorithm requires dimension reduction not greater than the total number of samples minus 1 , so $1 \leq \mathrm{LDA}$ dimension reduction $\leq 19$. There are 10 samples in each category, so $1 \leq k \leq 10$. Other parameters of MPCA are set to the optimized values.

4.1. Experiments on the ORL. The ORL face database contains a total of 400 images of 40 individuals (each individual has 10 gray scale images) [33]. Some photos are taken in different periods, and some are taken with the various countenances and the facial details. Each image is of a resolution of 256 grey levels per pixel [34]. Figure 4 shows image examples of two persons before preprocessing.

Now, images have been divided into three different groups. With the first group, we select the first 5 images of the first 20 persons as training data and the last 5 images of the first 20 persons as test samples for face identification. With the second group we select the first 5 images of the rest 20 persons as training data and the last 5 images of the rest 20 persons as testing samples. With the third group we select the first 5 images of 40 persons as training data and the last 5 images of 40 persons as testing samples.

Recognition error rate of PCA is shown in Figure 5.
Judging from the figure, when $k$ equals 1 , the recognition error rate reaches minimal value. PCA recognition accuracy reaches $58 \%-82 \%$.

Error rate of MPCA under different $k$ value is shown in Figure 6. As shown in the figure, when $k$ equals 1 , error rate is minimal. MPCA recognition accuracy reaches $75 \%-85 \%$ in the experiments.

When $k$ equals 8 , error rate of PCA + LDA algorithm reaches minimal value 7\%. How different LDA dimension reduction affects recognition accuracy is shown in Figure 7.

We can see from Figures 5, 6, and 7 that dimension after LDA increases as the number of samples also increases. When applying PCA + LDA algorithm, we use MPCA to decrease dimension of facial samples to 11 and then use LDA. For the 1 st group, reduce dimension to 7 . For the 2 nd group, reduce dimension to 8 . For the 3rd group, reduce dimension to 10 . We can conclude that the LDA algorithm is not satisfied with multidimensional objects. Accuracy of PCA + LDA reaches $86 \%-88 \%$.

MPCA + LDA algorithm only produces higher error rate of $10 \%-25 \%$ when $k$ equals 10 . In other situations, the recognition error rate is very low. When $k$ equals 8 , the error rate of different LDA dimensionality reduction is shown in Figure 8. Algorithm recognition accuracy rate reaches a high value.

Result of the experiments on ORL database is shown in Figure 9. Take recognition accuracy of four algorithms for comparison; the combination of MPCA and LDA does result in better recognition performance than traditional methods.

4.2. Experiments on More Face Databases. We choose FERET and YALE for our experiments. Implement steps are similar 


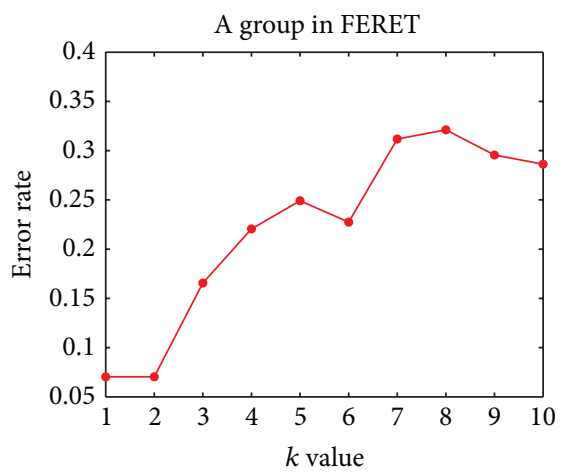

(a)

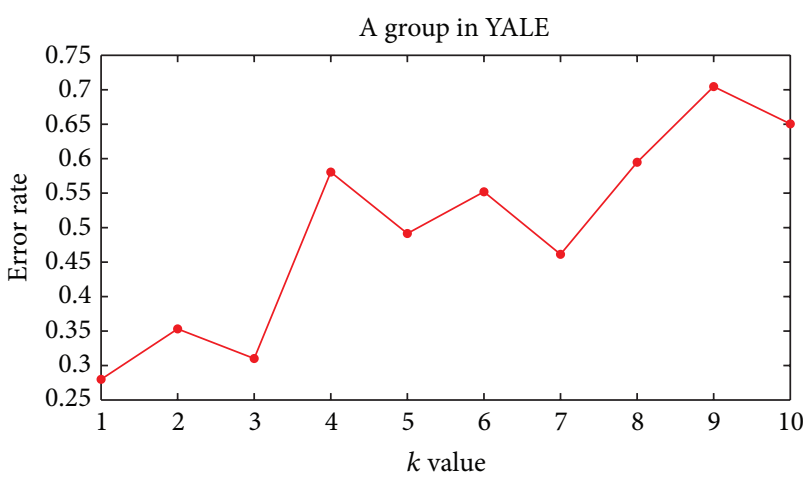

(b)

FIGURE 12: Recognition error rate of PCA against different $k$ values (best LDA dimension reduction). In one group of FERET, $k$ equals 27 . In one group of YALE, $k$ equals 63 .

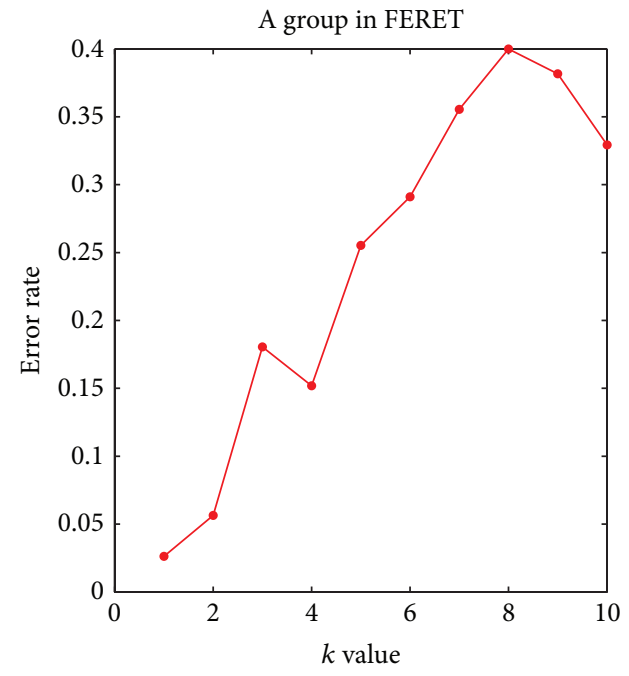

(a)

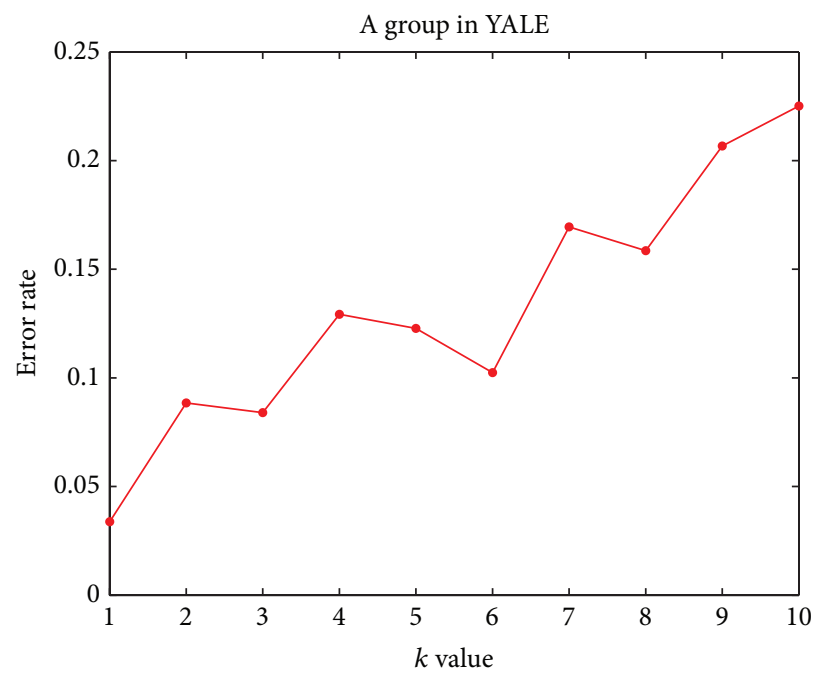

(b)

FIGURE 13: Recognition error rate of MPCA against different $k$ values.

to those in above section, so we just simplify steps and focus on the results.

FERET face database consists of a total of 1400 images of 200 individuals (each person has 7 different images). Figure 10 shows image examples of two persons before preprocessing.

YALE face database contains 165 images of 15 individuals; Figure 11 shows image examples of two persons before preprocessing [35].

The performance of PCA, MPCA, PCA plus LDA, and MPCA + LDA techniques is tested by varying the number of eigenvectors. We have chosen one group of result in each database for comparison.

PCA performed worse on YALE than on FERET because of the poor adaptability for the image brightness and facial expression, which is shown in Figure 12.

Though in Figure 13 MPCA performed much well on face recognition in YALE database, the process takes longer time than with PCA.
Figures 14 and 15 show that both PCA + LDA and MPCA + LDA can turn to high accuracy and low error rate in recognition. However, PCA + LDA effectively sees only the Euclidean structure, while MPCA + LDA successes to discover the underlying structure [36].

Compared against all the other algorithms, although with simple preprocessing, we can learn that MPCA + LDA has achieved best overall performance in both FERET and YALE databases.

\section{Conclusions}

This paper presents an algorithm for face recognition based on MPCA and LDA. As opposed to other traditional methods, our proposed algorithm treats data as multidimensional tensor and fully considers the spatial relationship. The advantage of our approach is of great relevance to applications and is capable of recognizing face dataset under different lighting 


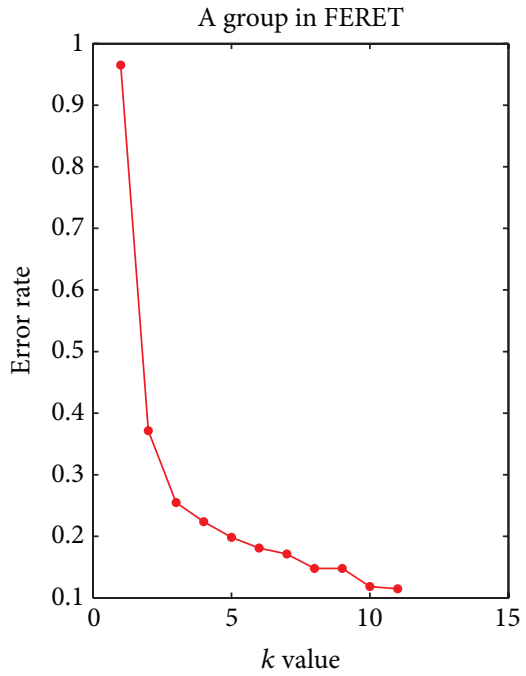

(a)

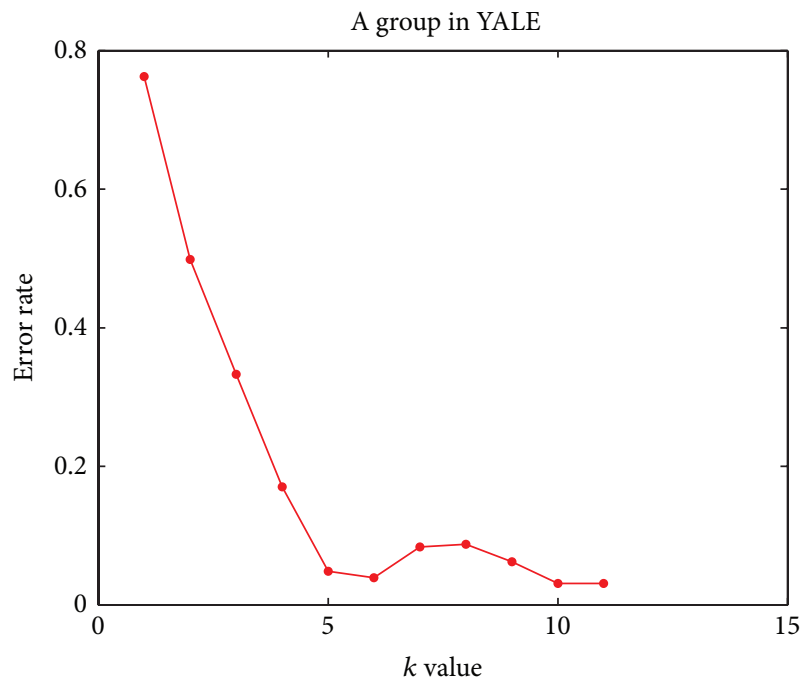

(b)

FIGURE 14: Recognition error rate of PCA + LDA against different LDA dimension reduction values.

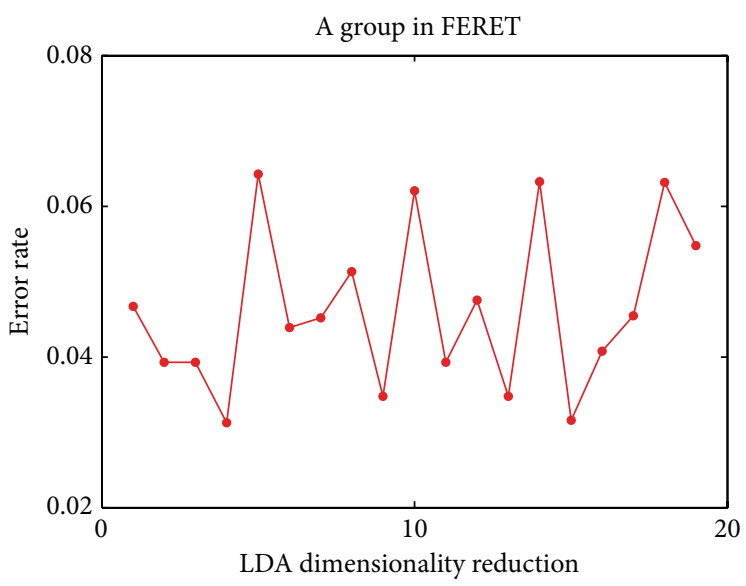

(a)

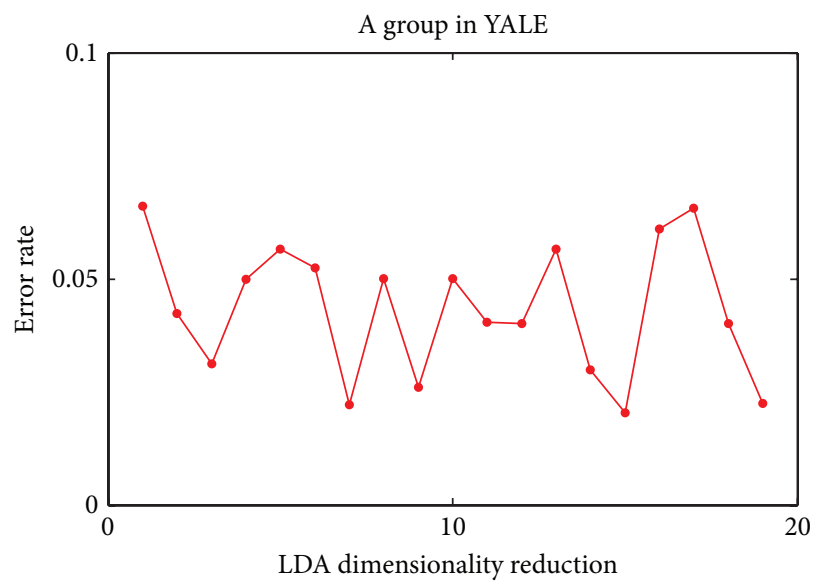

(b)

FIGURE 15: Error rate of MPCA + LDA algorithm against different LDA dimension reduction values.

conditions and with various facial expressions. LDA algorithm projects the data to a new space and has exact clustering result in our experiments. Compared with traditional face recognition algorithms, our proposed algorithm is not only a boost in recognition accuracy but also an unclogging of dimensionality bottlenecks and an efficient resolution of the small sample size problem. Future work of our research will include applying this approach on larger face databases such as on the CMU Multi-PIE, NIST's FRGC, and MBGC.

\section{Conflict of Interests}

The authors declared that there is no conflict of interests regarding the publication of this paper.

\section{Acknowledgments}

The authors would like to show an appreciation of reviewers' insightful and constructive comments and would like to thank everyone for their hard work on this research. The work was supported by a Grant from the Ph.D. Programs Foundation of Ministry of Education of China (no. 20120141120006), Hubei Planning Project of Research and Development (no. 2011BAB035), and Wuhan Planning Project of Science and Technology (no. 2013010501010146).

\section{References}

[1] F. Song, H. Liu, D. Zhang, and J. Yang, "A highly scalable incremental facial feature extraction method," Neurocomputing, vol. 71, no. 10-12, pp. 1883-1888, 2008. 
[2] W. Zhao, R. Chellappa, P. J. Phillips, and A. Rosenfeld, "Face recognition: a literature survey," ACM Computing Surveys, vol. 35, no. 4, pp. 399-458, 2003.

[3] H. Lu, K. N. Plataniotis, and A. N. Venetsanopoulos, "MPCA: multilinear principal component analysis of tensor objects," IEEE Transactions on Neural Networks, vol. 19, no. 1, pp. 18-39, 2008.

[4] S. Fernandes and J. Bala, "Performance analysis of PCA-based and LDA-based algorithms for face recognition," International Journal of Signal Processing Systems, vol. 1, no. 1, pp. 1-6, 2013.

[5] B. A. Draper, K. Baek, M. S. Bartlett, and J. R. Beveridge, "Recognizing faces with PCA and ICA," Computer Vision and Image Understanding, vol. 91, no. 1-2, pp. 115-137, 2003.

[6] K. Choudhary and N. Goel, "A review on face recognition techniques," in Proceedings of the International Conference on Communication and Electronics System Design, International Society for Optics and Photonics, 2013.

[7] R. Gottumukkal and V. K. Asari, "An improved face recognition technique based on modular PCA approach," Pattern Recognition Letters, vol. 25, no. 4, pp. 429-436, 2004.

[8] J. Li, B. Zhao, and H. Zhang, "Face recognition based on PCA and LDA combination feature extraction," in Proceedings of the 1st International Conference on Information Science and Engineering (ICISE '09), pp. 1240-1243, IEEE, December 2009.

[9] P. Viola and M. J. Jones, "Robust real-time face detection," International Journal of Computer Vision, vol. 57, no. 2, pp. 137154, 2004

[10] X. R. L. Y. Tang Liang, "An face recognition technique based on discriminative common vector in PCA transform space," Journal of Wuhan University, vol. 34, no. 4, 2009.

[11] H. Lu, K. N. Plataniotis, and A. N. Venetsanopoulos, "A survey of multilinear subspace learning for tensor data," Pattern Recognition, vol. 44, no. 7, pp. 1540-1551, 2011.

[12] A. K. Bansal and P. Chawla, "Performance evaluation of face recognition using PCA and N-PCA," International Journal of Computer Applications, vol. 76, no. 8, pp. 14-20, 2013.

[13] J. F. Pereira, R. M. Barreto, G. D. C. Cavalcanti, and T. I. Ren, "A robust feature extraction algorithm based on class-modular image principal component analysis for face verification," in Proceedings of the IEEE International Conference on Acoustics, Speech and Signal Processing (ICASSP '11), 2011.

[14] F. S. Tsai, "Dimensionality reduction for computer facial animation," Expert Systems with Applications, vol. 39, no. 5, pp. 49654971, 2012.

[15] H. Lu, K. N. Plataniotis, and A. N. Venetsanopoulos, "Uncorrelated multilinear discriminant analysis with regularization and aggregation for tensor object recognition," IEEE Transactions on Neural Networks, vol. 20, no. 1, pp. 103-123, 2009.

[16] W. Zhao, R. Chellappa, and N. Nandhakumar, "Empirical performance analysis of linear discriminant classifier," in Proceedings of the IEEE Computer Society Conference on Computer Vision and Pattern Recognition, pp. 164-169, IEEE, June 1998.

[17] Y. Xie, "LDA algorithm and its application to face recognition," Computer Engineering and Applications, vol. 46, no. 19, pp. 189192, 2010.

[18] S. Yan, D. Xu, Q. Yang, L. Zhang, and H. Zhang, "Multilinear discriminant analysis for face recognition," IEEE Transactions on Image Processing, vol. 16, no. 1, pp. 212-220, 2007.

[19] D. Zhang and Z. Zhou, "(2D)2 PCA: two-directional twodimensional PCA for efficient face representation and recognition," Neurocomputing, vol. 69, no. 1-3, pp. 224-231, 2005.
[20] D. Zhang, X. You, P. Wang, S. N. Yanushkevich, and Y. Y. Tang, "Facial biometrics using nontensor product wavelet and $2 \mathrm{~d}$ discriminant techniques," International Journal of Pattern Recognition and Artificial Intelligence, vol. 23, no. 3, pp. 521-543, 2009.

[21] J. Yang, D. Zhang, and A. F. Frangi, "Two-dimensional PCA: a new approach to appearance-based face representation and recognition," IEEE Transactions on Pattern Analysis and Machine Intelligence, vol. 26, no. 1, pp. 131-137, 2004.

[22] Y. Li, H. Xie, and Y. Zhou, "Study of eyebrow recognition based on 2 DPCA," Journal of Wuhan University, vol. 57, no. 6, pp. 517$522,2011$.

[23] H. Lu, K. N. Plataniotis, and A. N. Venetsanopoulos, "Uncorrelated multilinear principal component analysis through successive variance maximization," in Proceedings of the 25th International Conference on Machine Learning, pp. 616-623, July 2008.

[24] L. De Lathauwer, B. De Moor, and J. Vandewalle, "On the best rank-1 and rank- $\left(R_{1} R_{2}, \ldots, R_{n}\right)$ approximation of higher-order tensors," SIAM Journal on Matrix Analysis and Applications, vol. 21, no. 4, pp. 1324-1342, 2000.

[25] C. Chen, S. Zhang, and Y. Chen, "Face recognition based on MPCA," in Proceedings of the 2nd International Conference on Industrial Mechatronics and Automation (ICIMA '10), pp. 322325, Wuhan, China, May 2010.

[26] P. N. Belhumeur, J. P. Hespanha, and D. J. Kriegman, "Eigenfaces versus fisherfaces: recognition using class specific linear projection," IEEE Transactions on Pattern Analysis and Machine Intelligence, vol. 19, no. 7, pp. 711-720, 1997.

[27] X. Su, Q. Zeng, and X. Wang, "Several combination methods of face recognition based on PCA and LDA," Computer Engineering and Design, vol. 33, no. 9, pp. 3574-3578, 2012.

[28] G.-F. Lu, Y. J. Wang, and J. Zou, "Improved complete neighbourhood preserving embedding for face recognition," IET Computer Vision, vol. 7, no. 1, pp. 71-79, 2013.

[29] E. Nasibov and C. Kandemir-Cavas, "Efficiency analysis of KNN and minimum distance-based classifiers in enzyme family prediction," Computational Biology and Chemistry, vol. 33, no. 6, pp. 461-464, 2009.

[30] J. Shermina, "Face recognition system using multilinear principal component analysis and locality preserving projection," in Proceedings of the IEEE GCC Conference and Exhibition (GCC '11), pp. 283-286, IEEE, February 2011.

[31] Y. Liaw, M. Leou, and C. Wu, "Fast exact k nearest neighbors search using an orthogonal search tree," Pattern Recognition, vol. 43, no. 6, pp. 2351-2358, 2010.

[32] F. S. Samaria and A. C. Harter, "Parameterisation of a stochastic model for human face identification," in Proceedings of the 2nd IEEE Workshop on Applications of Computer Vision, pp. 138-142, Sarasota, Fla, USA, December 1994.

[33] Y. Jin and Q. Ruan, "Orthogonal locality sensitive discriminant analysis for face recognition," Journal of Information Science and Engineering, vol. 25, no. 2, pp. 419-433, 2009.

[34] P. P. Paul and M. Gavrilova, "Multimodal cancelable biometrics," in Proceedings of the IEEE 11th International Conference on Cognitive Informatics \& Cognitive Computing (ICCI '12), 2012.

[35] P. Punitha and D. S. Guru, "Symbolic image indexing and retrieval by spatial similarity: an approach based on B-tree," Pattern Recognition, vol. 41, no. 6, pp. 2068-2085, 2008.

[36] X. He, S. Yan, Y. Hu, P. Niyogi, and H. Zhang, "Face recognition using Laplacianfaces," IEEE Transactions on Pattern Analysis and Machine Intelligence, vol. 27, no. 3, pp. 328-340, 2005. 


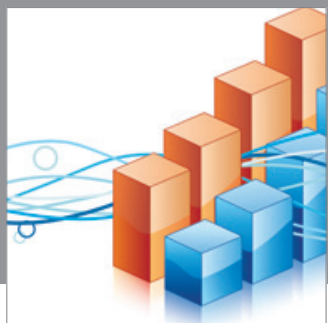

Advances in

Operations Research

mansans

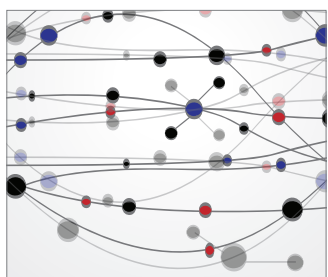

The Scientific World Journal
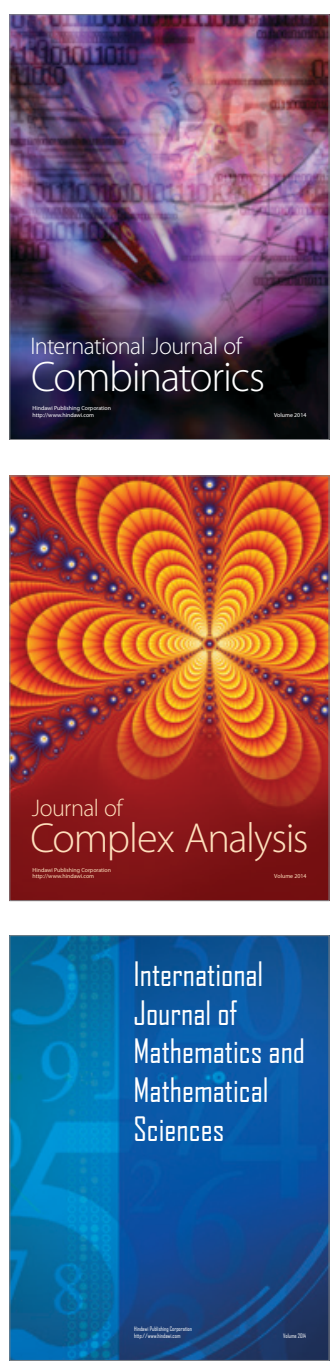
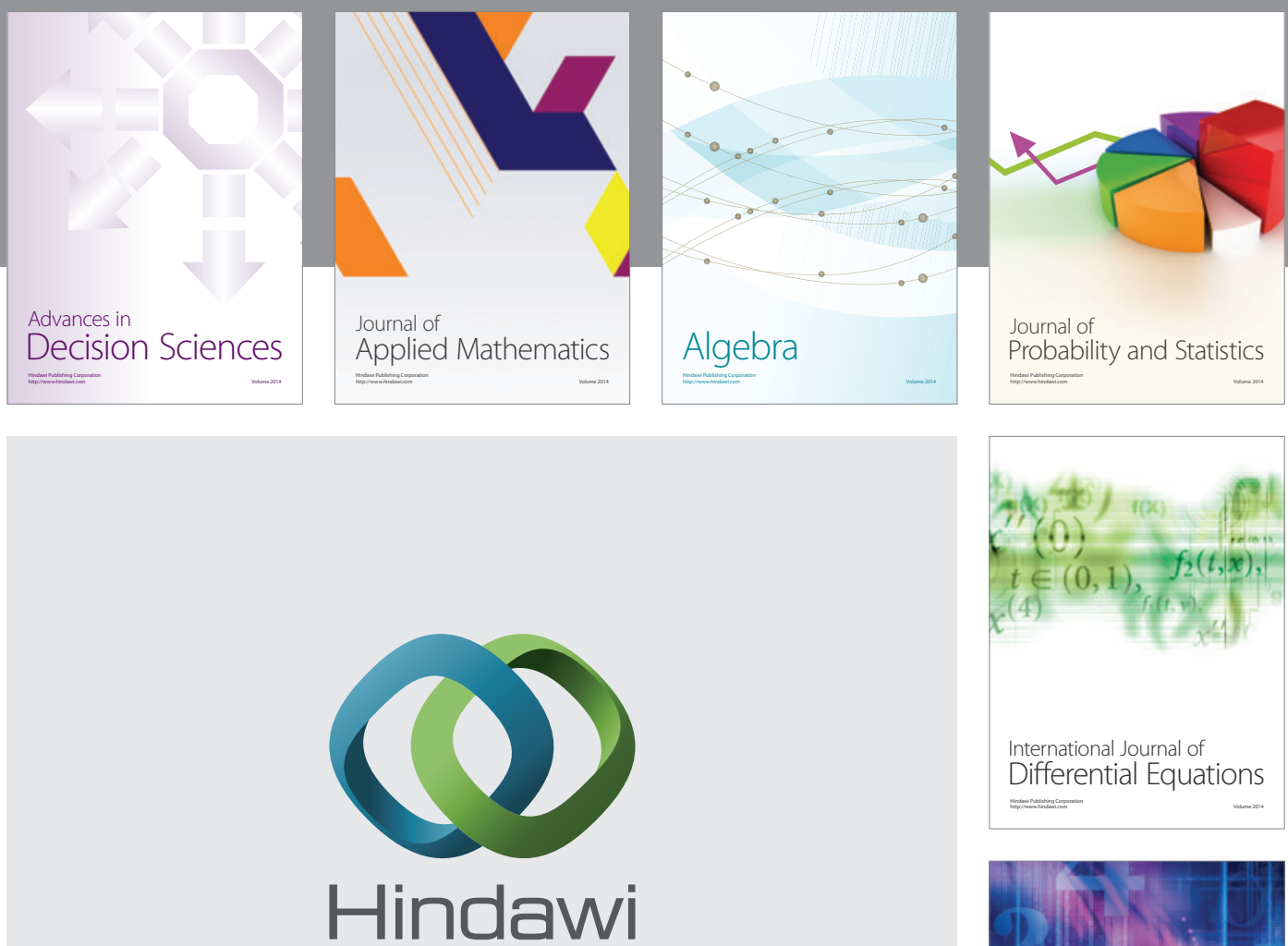

Submit your manuscripts at http://www.hindawi.com
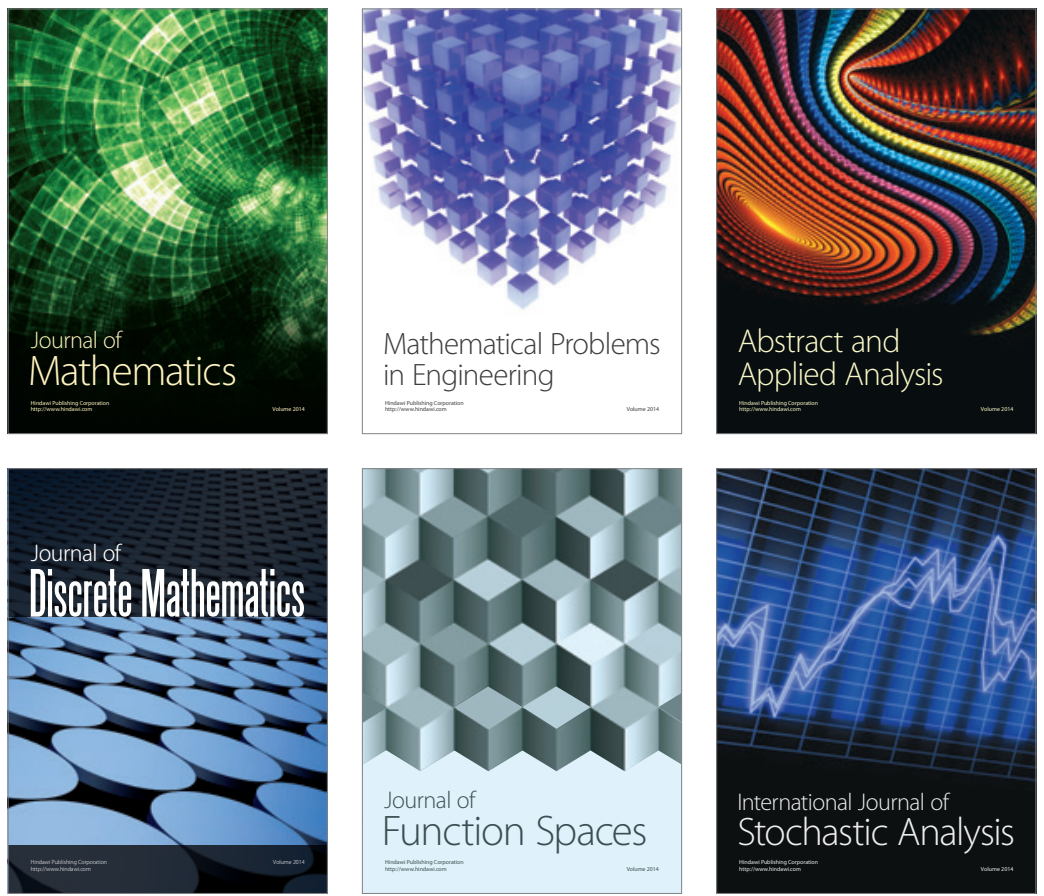

Journal of

Function Spaces

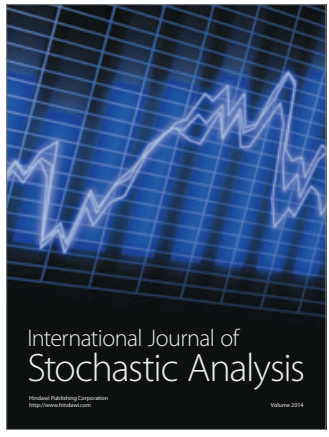

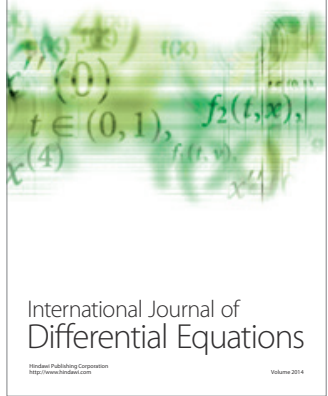
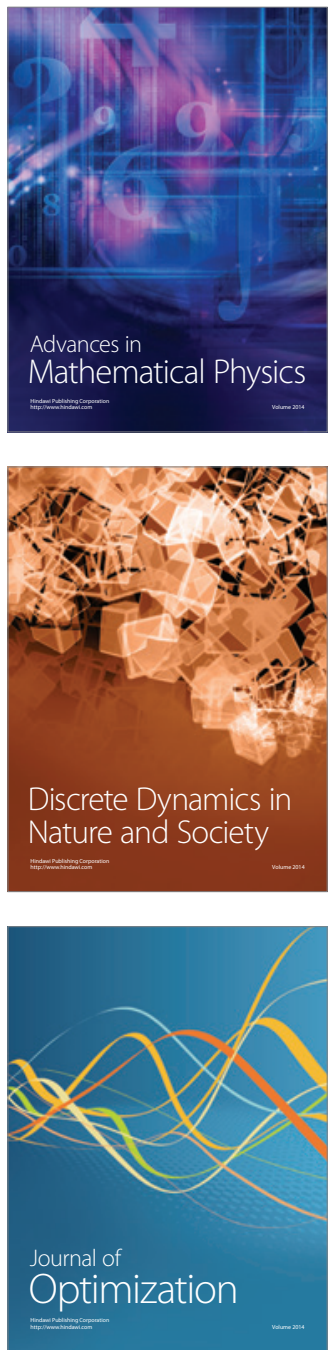